\title{
Real-time threat evaluation in a ground based air defence environment
}

\author{
JN Roux* JH van Vuuren ${ }^{\dagger}$
}

Received: 15 December 2007; Revised: 19 March 2008; Accepted: 1 April 2008

\begin{abstract}
In a military environment a ground based air defence operator is required to evaluate the tactical situation in real-time and protect Defended Assets (DAs) on the ground against aerial threats by assigning available Weapon Systems (WSs) to engage enemy aircraft. Since this aerial environment requires rapid operational planning and decision making in stress situations, the associated responsibilities are typically divided between a number of operators and computerized systems that aid these operators during the decision making processes. One such a Decision Support System (DSS), a threat evaluation and weapon assignment system, assigns threat values to aircraft (with respect to DAs) in real-time and uses these values to propose possible engagements of observed enemy aircraft by anti-aircraft WSs. In this paper a design of the threat evaluation part of such a DSS is put forward. The design follows the structured approach suggested in [ROUX JN \& VAN VUUREN JH, 2007, Threat evaluation and weapon assignment decision support: A review of the state of the art, ORiON, 23(2), pp.151-187], phasing in a suite of increasingly complex qualitative and quantitative model components as more (reliable) data become available.
\end{abstract}

Key words: Threat evaluation, decision support, air defence, aircraft.

\section{Historical Background}

The story of modern warfare begins with Napoleon. His considerable impact on the art of war is still felt today in a variety of ways - his innovative genius transformed strategy, tactics, organization and logistics. In particular, air warfare is said to have had its origin in Napoleon's balloon corps at the end of the 18th century and its infancy in the various schemes for using balloons and airships for reconnaissance which were put forward during the 19 th century.

Germany's World War I zeppelins, which conducted cross-channel bombing attacks against London, preceded the airplane as the first active aerial threat. These early airships could

\footnotetext{
${ }^{*}$ Corresponding author: Department of Logistics, University of Stellenbosch, Private Bag X1, Matieland, 7602, South Africa, email: jroux@rrs.co.za

${ }^{\dagger}$ Department of Logistics, University of Stellenbosch, Private Bag X1, Matieland, 7602, South Africa.
} 
not only navigate, but could also carry enough bombs to cause considerable damage. However, the zeppelins would prove to have an insurmountable vulnerability to incendiary weapons and would be relegated back to use as passive aerial weapons after 1918, and then only rarely: The airplane proved to be the aerial threat of the future, as well as the cause of the zeppelins' subsequent demise [16].

The first flight by the Wright brothers [26] in 1903 changed the way wars would be fought forever, although this was not immediately obvious. Powered flight altered the very nature of the aerial threat that had existed up to that point from a passive one (observation) to an active one (bombing and strafing). The actual use of aircraft for military purposes began in a tentative fashion in 1911 when the Italian Army embraced the field of military aviation. The Italian Army Aeronautical Service was formed in 1884 and balloons were used for reconnaissance purposes during the Eritrean War of 1887/88 [28]. In 1911 five aircraft and two small airships were employed (by the Italians) in army manoeuvres in Libya with moderate success.

In the Balkan War of 1912 [27, 36], the Bulgarians used a number of aircraft flown by private owners anxious to assist the army. One pilot, named Constantin, was in the process of making an aerial study of the Turkish lines at Chataja when he was struck and killed by a rifle bullet from the ground. The unfortunate Constantin thus has the melancholy distinction of being the first aviator known to be killed by anti-aircraft fire.

During the first world war the novel emergence of an active air threat [11] necessitated the development of countermeasures - among them fighter aircraft and anti-aircraft artillery. With Hitler's elevation to power in 1933, Britain's objective of maintaining the balance of power in Europe came under considerable pressure. Countering the German air threat required an almost impossible expansion of the Royal Air Force. This led to the establishment of a team of scientists that would 'consider how far recent advances in scientific and technical knowledge can be used to strengthen the present methods of defence against hostile aircraft' [23]. These activities led to the design and development of radar for Air Defence $(\mathrm{AD})^{1}$ in Britain and marks the first Operations Research success story. These scientists, using mathematical techniques, developed methodologies that could be used for successful interception of enemy aircraft. Together with service personnel of the Royal Air Force they ensured that Britain possessed a technically efficient and fully operational early warning system of aircraft interception along the greater part of its vulnerable south and east coasts. Here service personnel refers to women in the military who moved icons around a large map, based on radar reports and whose work was used by commanders to make decisions about assigning interceptors against bombers. This is seen as one of the first Threat Evaluation (TE) Decision Support Systems (DSSs).

\section{Emerging theories of warfare}

The continual and rapid change in technology portrayed briefly in $\S 1$ forced military researchers into the development of new theories of warfare, where shared information, co-

\footnotetext{
${ }^{1}$ The meanings of all military acronyms used in this paper are summarized in an appendix following directly on the bibliography at the end of the paper.
} 
operation between, and command and control of all forces, regardless of their operational environment, are managed. As a result, more and more military systems are becoming automated today, not only to decrease operator workload, but also to facilitate tactical operations previously not possible and to increase human survivability and safety during volatile combat situations [33]. Examples of such systems include the introduction of unmanned aerial vehicles [17, 29], unmanned combat aerial vehicles [48] and combat robots $[24,41]$.

One of the noteworthy emerging theories of warfare in the current information age is network centric warfare (NCW) [33, §7]. According to Raduege [30] "... network centric warfare is not just about technology; it is an emerging theory of war and the next art and science of warfare to be exploited." The notion of NCW is a derivative of network centric computing. The evolution of computing from platform centric computing to network centric computing has largely been enabled by recent key developments in information technology, making it much easier for computers with different operating systems to interact with each other. Network centric computing is currently being exploited by early adopters to provide a competitive edge in the commercial business sector. Similarly, the emerging concepts of NCW exploit information superiority to provide a competitive edge in warfare [37].

NCW is not all about networks, rather it is about how wars are fought and how power is developed. During the industrial age power came from mass [2], but in the twentyfirst century power tends to come from information, access and speed [6]. According to Cebrowski [6], "... we are witnessing important, though nascent changes emerging in the realm of sensors and how they will be used on future battlefields. In Operation Enduring Freedom in Afghanistan we are looking for future implications. We are seeing warfare dominated more by sensors than perhaps any other piece of equipment. The ability to sense the environment, to sense the enemy and to be networked enough to transmit that critical data to all who require it, is a trend line emerging from current operations. The issue is not weapons reach. The issue is sensor reach." Weapon systems (WSs) have advanced to such an extent that if military systems are able to detect a target, the probability of killing or destroying it is extremely high. Consequently, potential enemies are working very hard to make it difficult for adversaries to sense their targets - warfare is thus clearly shifting from a weapons game to a sensor game.

It is this overwhelming amount of sensor and other information available due to the battle for information superiority that necessitates better situation awareness [33, $\S 5]$ and DSSs to aid military operators and commanders responsible for critical real-time decision making. Hence, there is also a need for TE systems which are able to take all available variables (kinematic and non-kinematic data) into account and assess enemy behaviour; resulting not only in supportive data (e.g. a prioritized list of threats), but in some cases also in the triggering of automatic countermeasures against the threats. The automation of TE is a complex (and relatively new) problem in military science [33, §6.2].

Military art is in many ways the centerpiece of military science. In it one studies the specifics of combat, and attempts to reduce the many factors to a set of principles that govern all interactions of the field of battle. As such, it directs the planning and execution of battles, operations, and wars as a whole. Broadly speaking, there are two major systems 
of military art [45]: (1) the "Western" system and (2) the "Russian" system. Each system reflects and supports strengths and weakness in its underlying society. Generally, "Western" societies have higher levels of education and technology. In contrast, third-world societies (which are based on the Russian system) have lower levels of education and technology, but have much more raw manpower in their military than Western societies are willing (or able) to devote. Typically, the foundation (or starting point) for research on TE systems focuses on the similarities between these two military arts, necessitating a generic model and general principles for TE. On implementation level, it is obvious that customization of the system according to the user's doctrine and capabilities, as well as the enemies' predicted doctrine and capabilities, is required. Doctrine may be described by the analysis component provided in military art [45]. Prediction of enemy doctrine and capabilities is a very cumbersome process, since behaviour regarding attack techniques and force movement strategies are typically kept secret. Broadly speaking, doctrinal behaviour may be classified as either Western, Soviet or Chinese [45].

By examining these three general doctrinal classifications, the complexity of a general TE solution is evident. The solution is further complicated by the fact that enemy units may not always obey commands, or even worse, that the units may behave in a completely unexpected way, driven by the fear of losing their lives. Other cognitive factor anomalies exist, moving into the fourth generation of warfare [40], where enemy strategy is driven by "unfair" or "irrational" actions. "Roughly speaking, the fourth generation of warfare includes all forms of conflict in which the other side refuses to stand up and fight fair $[s i c]$. What distinguishes the fourth generation of warfare from earlier generations is that typically at least one side is something other than a military force organised and operating under the control of a national government, and one that often transcends national boundaries" [13].

\section{Threat evaluation in context}

Informally, the purpose of a TE DSS is to rank observed enemy craft according their threatening behaviour with respect to a number of Defended Assets (DAs). A theoretical background on the TE process with regards to command and control, data fusion, situation awareness, Decision Support (DS) and NCW is provided in [33]. In theory, it is evident that the TE process provides DS (which improves command and control as well as situation awareness) and is dedicated to improving the operational tempo of operators.

For the purposes of this paper, we shall focus on Ground Based Air Defence System (GBADS) battery level TE, purely in an attempt to render the TE elements that have to be considered to some manageable number. However, many of the concepts and modelling techniques introduced in the remainder of this paper may be generalised to TE processes on other levels of $\mathrm{AD}$ or in domains other than AD. On a GBADS battery level, a small number of DAs are typically protected by a limited number of ground based WSs and sensor systems. Furthermore, AD control is typically directed by an air picture manager and Fire Control Officer (FCO) - responsible for Threat Evaluation and Weapon Assignment (TEWA) on the battery level (see, for example, $[33, \S 6.4]$ ).

The remainder of this paper is organised as follows. In $\S 4$ we review the various elements 
of a TE DSS, such as the tactical environment, possible threats to the system, sensors employed by the system, assets typically defended by the system and the role of operators with respect to the system. This is followed, in $\S 5$, by a detailed TE DSS design proposal, including a suite of TE models which may be implemented on three levels of increasing complexity (which are meant to run concurrently, complementing each other with respect to DS in real time). Finally, a number of practical TE DSS implementation thoughts are given in $\S 6$, after which the paper closes, in $\S 7$, with some concluding remarks.

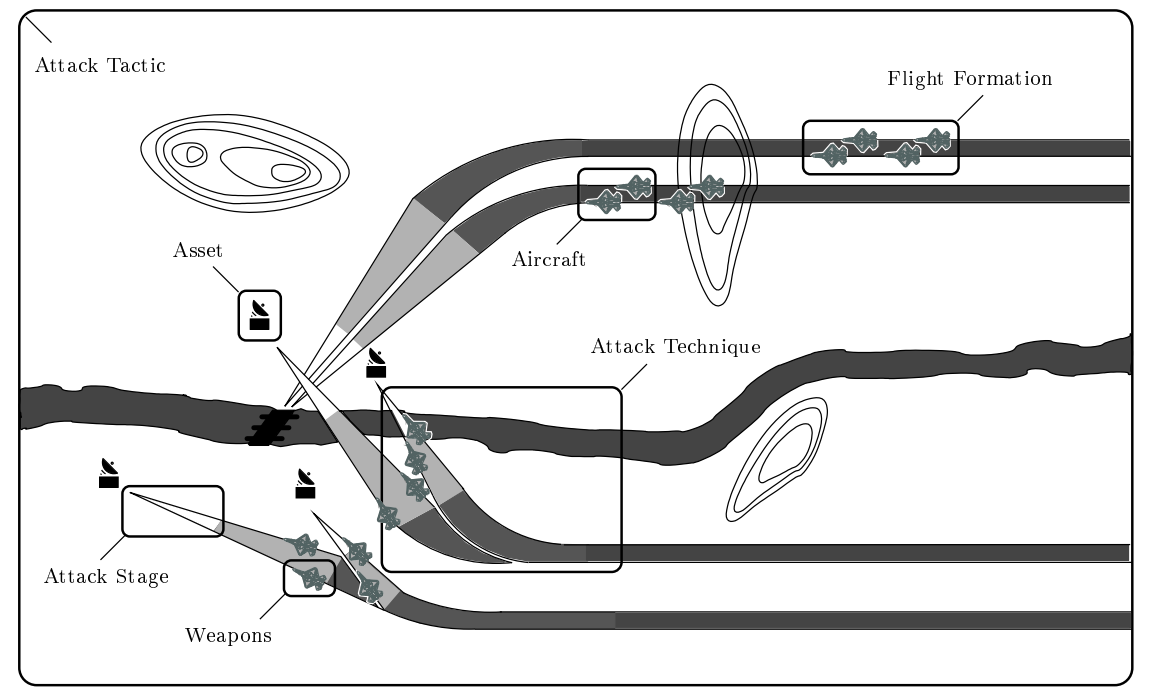

Figure 1: The various formative elements of a tactical situation (as seen from above).

\section{Elements of a threat evaluation decision support system}

The elements of a TE DSS may be identified by considering a typical AD scenario (see Figure 1). When observing an area of responsibility within a tactical environment, a typical attack is assumed to consist of an enemy pilot flying a specific aircraft (threat) to complete a specific attack technique (profile) in order to deliver a specific weapon so as to damage or destroy a specific asset. These co-ordinated actions are typically stated by a pre-defined tactic, determined by the enemy commander. Where multiple aircraft are considered, specific flight formations are used. Of course, the aerial threats are observed by the sensor operators using various types of sensors. Let us now consider the various TE elements identified above in some detail.

\subsection{The tactical environment}

The tactical environment is the physical volume within which TE takes place and hence influences a number of choices regarding the physical elements it encompasses. The tactical environment may include air, land, surface (which refers to the surfaces of oceans, rivers, dams and lakes), subsurface and potentially even space.

Considering the joint $\mathrm{AD}$ operations of the emerging theories of warfare introduced in 
$\S 2$ and $\S 3$, it is evident that a thorough investigation of all applicable environments is required during the pre-deployment phase of a mission. Furthermore, the modelling of these threat environments is of significant importance for TE purposes, since terrain types and weather conditions in particular may bear a strong influence on the deployment of AD systems, the accuracy of sensors, the capabilities of WSs and the enemy's choice of attack craft and profiles.

\subsection{Threats to the system}

As mentioned, only enemy craft in the aerial environment (see $\S 4.1$ ) are considered threats to the system in this paper. These threats are very manoeuvrable and their aerodynamic kinematics exhibit six degrees of freedom. The threatening manoeuvres exhibited or attack techniques adopted by these aircraft are typically restricted by obstructions in the land environment and strongly depend on craft and pilot capabilities.

The choice of system threats is not trivial, since each category of threats embodies a variety of craft and weapon characteristics, including attack speed, attack density, effective range, typical missions (objectives) and targets, weapons carried (i.e. ordnance, armament), tactics, radar signature and physical size, environmental constraints, and economics. The various categories of threats encountered in an aerial environment typically include missiles, electronic warfare platforms, unmanned aircraft and manned aircraft.

Furthermore, a number of factors may influence the enemy's choice of aircraft, weapon type or attack technique, which may consequently differ for the same kind of mission. These factors include minimizing the cost of the mission, maximizing human and equipment survivability, adhering to the rules of engagement, following doctrine, recalling similar previous encounters and even implementing personal preferences of the general in command [33, $\S 6.4]$. Own force dependency on the above mentioned factors may be problematic, since enemy manoeuvre and doctrine data are near impossible to obtain - yet the accuracy of TE model results usually depend heavily on data quality and availability.

\subsubsection{Aircraft type}

The complexity surrounding the analysis of a system threat category suggests the desirability of commencing research on a small subset of threats. Consequently, only fixed wing aircraft are considered in this paper. Fixed wing aircraft may be divided into three broad categories: fighter/bomber, reconnaissance and transport/tankers. Since the latter two categories pose no direct threat to the DAs, they are ignored in this paper.

\subsubsection{Weapon type}

Weapons refer to the armament used by the enemy aircraft during the engagement of DAs. Armament for fixed wing aircraft may be divided into a number of categories, delivered by increasingly complex and ever evolving weapon delivery systems. The choice of weapon type depends on various factors, such as the effectiveness of the weapon with respect to the DA and the operational value of the DA attacked. Engagement by the attacking aircraft is also influenced by the type and size of the DA. Generally the following 
weapon categories are used against various assets: guns/cannons, rockets, guided missiles, guided ballistic bombs, free falling bombs, braked bombs, container/cluster bombs and fire bombs. Note that electronic warfare weapons are excluded from this list. Some of the categories described above also contain stand-off weapons. As soon as the stand-off weapon is released, the missile becomes a threat of its own accord and the aircraft may still be evaluated as a separate threat, depending on its remaining armament and subsequent behaviour.

\subsubsection{Technique type}

The movement of an aircraft during an engagement may typically be described by means of any of a number of profiles. These profiles are determined by kinematic parameters and manoeuvre capabilities, and research in this area contributes in a significant manner to the accuracy of a TE system. These profiles are not always pre-defined and in many cases progression towards a starting point of an attack profile may be determined by the pilot in real-time, by finding the easiest or safest path. We divide aircraft flight techniques into two general categories: (1) approach techniques and (2) attack techniques. The analysis of the attack technique is of extreme importance for a TE system. However, attack technique data are especially hard to come by, since they depend on a variety of factors, including meteorological conditions, the specific aircraft used, the weapon(s) delivered, the country of origin (dictated by its doctrine and rules of engagement), and the proficiency and preferences of the pilot.

Although techniques differ greatly, a number of general similarities exist between these techniques and hence several generalized profiles may be identified. Examples include the combat hump dive (pitch and dive), the high level dive, the combat turn dive, tossbombing and low level attack techniques. We divide every attack technique into four phases: (1) the approach phase during which an aircraft travels to a pre-determined location to commence execution of its attack technique, (2) the manoeuvre phase, which usually represents the manoeuvres required to execute a specific attack technique, (3) the attack phase, typically encompassing the stabilization, aiming and weapon release stages of an engagement, and (4) the getaway phase during which the aircraft travels away from the asset after engagement, attempting to avoid a counter strike. Note that we divide the attack phase further into a number of stages (stabilization, aiming and weapon release) which are the smallest distinguishable and independent behavioural building blocks of an aircraft flight technique. An example of a combat hump dive, together with its subdivision into attack phases and stages, is shown in Figure 2.

\subsubsection{Flight formations}

A formation of aircraft may be seen as a single threat if not distinguishable by the available sensors and hence may be described by the same mathematical means as for a single threatening aircraft. Of course, the threats that these formations hold for assets differ from those of single aircraft, but it is assumed that aircraft in the same formation will complete the same attack technique and will deliver the same weapons. Clearly, a number of aircraft (raid size) property and the capability of the radar sensors to identify aircraft 


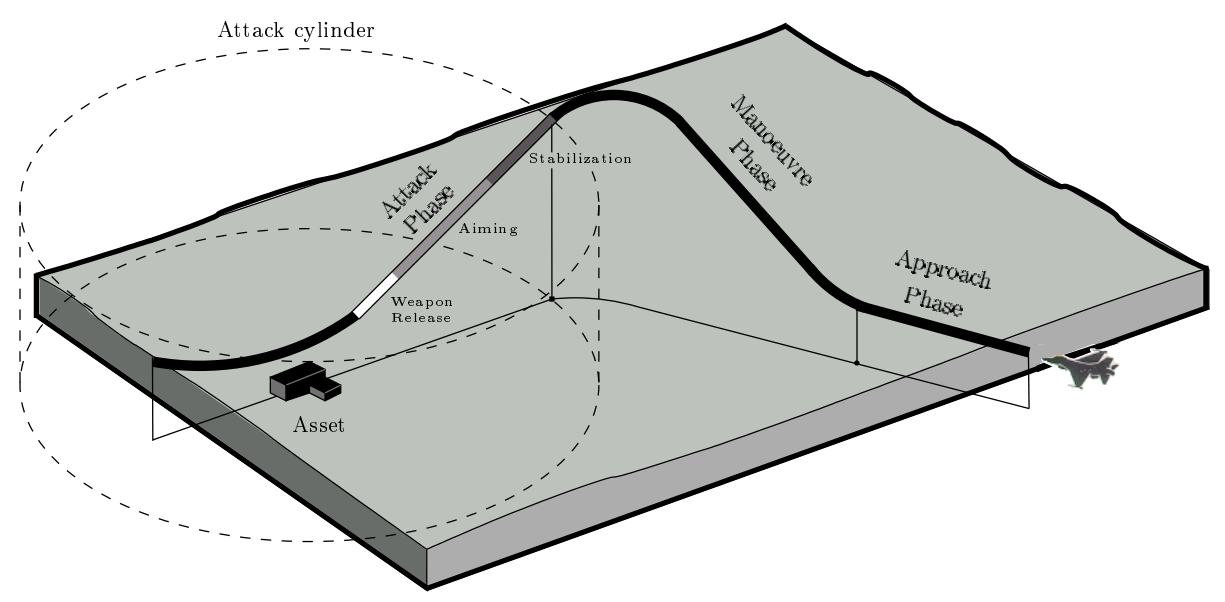

Figure 2: The combat hump dive attack technique.

formations (and their properties) should be in place so as to render the evaluation of aircraft formations possible. A thorough understanding of the forming and behaviour of formations is essential when scenarios are developed for testing purposes of a TE system.

\subsubsection{Attack tactics}

Before any mission is executed, the tactics are planned to the finest detail. Decisions depend on a number of factors, including meteorological conditions, doctrine, anti-aircraft defences expected, target characteristics, etc. Since the number of different situations is very large, specific tactics are not considered in this paper.

\subsection{Sensors}

Input to the TE process is received from a separate track management process which fuses sensor tracks received from various sensor systems as part of the sensing process [33, $\S 8]$. The accuracy and update rate (quality and quantity) of sensor data bear a very strong influence on the TE results, especially where more complex TE models (i.e. probabilitybased TE models) are used [32]. Furthermore, for the purposes of early warning (i.e. procuring more time for FCO decisions and actions), the effective detection range of the sensors is also important.

In a network centric $\mathrm{AD}$ environment, sensors may include various types ${ }^{2}$, such as $[15,46]$ : thermal (e.g. infra-red sensors), electromagnetic (e.g. radar), mechanical (e.g. position sensor), optical radiation (e.g. light sensors) and acoustic (e.g. sonar) sensors. Within the context of a GBADS TE system, the conventional sensor system used is a 2D surveillance (search) radar system. An example of typical specifications for such a system is shown in Table 1.

Nevertheless, within the modern day GBADS context, the capability of all available sensors

\footnotetext{
${ }^{2}$ Since sensors function by means of an exchange of energy, they may be classified according to the type of energy transfer they detect [47].
} 


\begin{tabular}{|c|c|c|c|c|}
\hline \multirow{4}{*}{$\begin{array}{r}\text { Long Range } \\
\text { Medium Range } \\
\text { Short Range }\end{array}$} & Update Rate & Range & Range Accuracy & Azimuth Accuracy \\
\hline & $10 \mathrm{sec}$ & $200+\mathrm{km}$ & $100-500 \mathrm{~m}$ & $0.5-2 \mathrm{deg}$ \\
\hline & $2-4 \mathrm{sec}$ & $50-200 \mathrm{~km}$ & $20-100 \mathrm{~m}$ & $0.1-0.5 \mathrm{deg}$ \\
\hline & $1 \mathrm{sec}$ or better & $0-50 \mathrm{~km}$ & $5-20 \mathrm{~m}$ & $0.1 \mathrm{deg}$ \\
\hline
\end{tabular}

Table 1: An example of typical 2D surveillance radar specifications [50].

are of importance. A wide variety of sensors are available. When choosing sensors, the trade-off between effective range, and accuracy and update rate should be borne in mind. $3 \mathrm{D}$ surveillance sensors add the third dimension of elevation to TE input data. For these sensors, elevation accuracy varies between 0.1 and 5 degrees and elevation angles (for detection, where ground level is at 0 degrees) of 0-30 degrees are common, angles of 30-75 degrees are less common and angles of above 75 degrees are only covered in exceptional cases [50]. Furthermore, for short to medium range sensors, threats may be detected up to an altitude of 2000-3500 metres; for longer range sensors threats may be detected up to an altitude of 30000 metres in some cases $^{3}$ [50].

Although not often used on long range surveillance radar, the doppler effect ${ }^{4}$ may be used to measure the radial velocity of a target. This effect is also used in tracking radar radar sensors dedicated to track a single or small number of targets at relatively small effective ranges (e.g. ranges of 15-20 kilometres). These systems are characterised by their high level of accuracy (e.g. range and azimuth accuracies of 5 metre and 1 milli-radian respectively) and their high update rate (e.g. an update rate of 20 milli-seconds). Note that some multi-function radar systems also have both search and tracking capabilities.

In addition to searching and tracking of targets, the target radar signature may be used for hostility and type (platform) classification of the target - another very important input to the TE system. Typical techniques used are non-cooperative target recognition techniques $[9,10]$ (based on high range resolution radar profiles and inverse synthetic aperture radar images [10]) and jet engine modulation ${ }^{5}$ based target identification techniques. However, these techniques are typically used for short range surveillance or in tracking radars and characteristically require long target dwell times.

The quality of data not only depends on the type of radar, but may deteriorate because of internal or external interferences caused by unwanted signals ${ }^{6}$. Inferences may be caused internally by signal noise, and externally by clutter (e.g. actual radio frequency echoes returned from other targets, multipath echoes from the related target due to ground reflection, atmospheric ducting or ionospheric reflection/refraction) and jamming [46]. The use of diverse sensors may reduce the above mentioned sensor data inaccuracies via data fusion techniques (as part of the track management process [33, §8]) when system tracks are formed.

For the purposes of this paper, the term aircraft attributes refers to the various factors or variables necessary for efficient evaluation of aircraft behaviour. For convenience of

\footnotetext{
${ }^{3}$ Note the large difference in dimensions between the range and altitude capabilities of radar.

4 "The Doppler effect, named after Christian Doppler, is the change in frequency and wavelength of a wave as perceived by an observer moving relative to the source of the waves" [43].

${ }^{5}$ The jet engine modulation phenomenon is caused by radar returns from the rotating structure of jet engines [4].

6 "The ability of the radar system to overcome unwanted signals defines its signal-to-noise ratio" [46].
} 
mathematical treatment and overall model clarity, we distinguish between two classes of aircraft attributes, namely measured attributes and derived attributes.

The values of measured attributes are obtained from sensor systems, such as radar sensors, observing the tactical environment in real-time. These measured attributes are aircraft specific, and where multiple radar sensors are used, their values are typically obtained using data fusion techniques. For the purposes of this paper, computation of these attributes are assumed to form part of the track management process [33, §8]. Measured attributes depend on two parameters: (1) an index or identification number allocated to the relevant observed aircraft and (2) a timestamp related to the value measured. Examples of these attributes include altitude, position, speed and course (see Figure 3).

Derived attributes, on the other hand, are computed from combinations of measured aircraft attributes with respect to a specific asset. The latest (real-time) value of a combination of measured attributes at a specific time (and possibly a number of instances of their history values) are used for their computation. Hence, these attributes depend on four parameters: (1) the index or identification number of the observed aircraft, (2) an index or identification number allocated to the DA, (3) the related timestamp and (4) a number of history values. Examples of derived attributes include range to the DA and bearing with respect to the DA (again, see Figure 3).

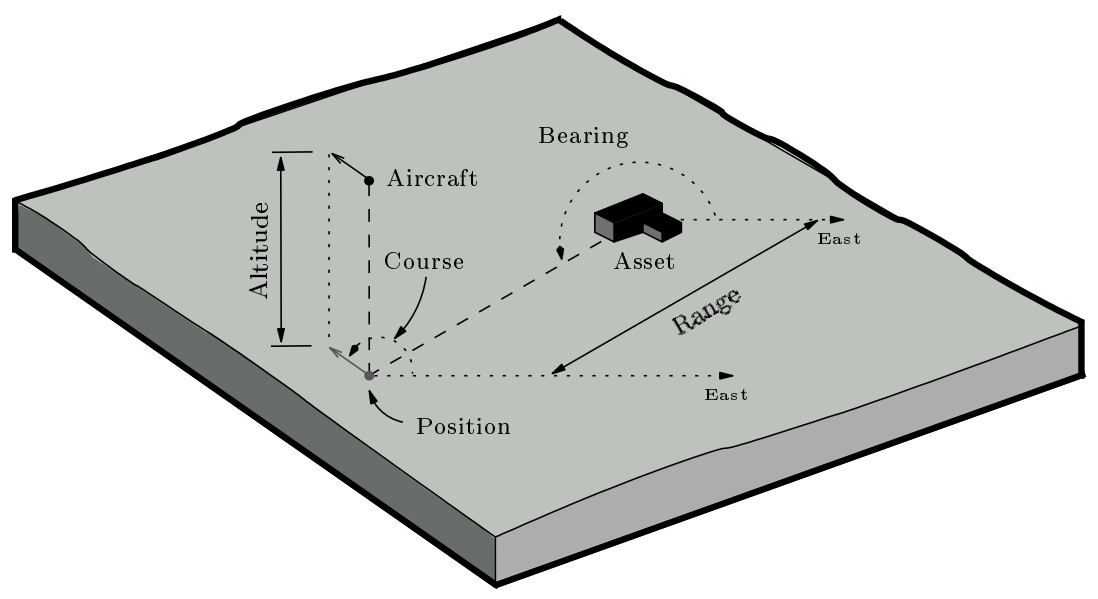

Figure 3: Various aircraft attributes.

\subsection{Defended assets}

Ground based assets may be divided into the following types [39, 42]: air bases (including runways, aircraft protected by shelters, unprotected aircraft, fuel depots, hangars, buildings, ammunition depots), oil refineries (including fuel tanks and associated installations), railway installations (such as rail tracks, trains, signal boxes, buildings), harbours (including ships and associated installations), crossroads, bridges (including main bridges and combat bridges), power plants, factories, field fortifications, command posts, gun and troop emplacements, anti-aircraft defences, early warning and allocation radars, soft skinned vehicle accumulations and armoured vehicle formations. 
DAs are identified from the set of all assets under the commander's control. A reduction in the number of assets that require defence during TE from the total number of assets is critical, since the time required to execute TE processes should be reduced so as to maximize operator decision/reaction time (i.e. increasing the operational tempo [33, §9]), and more importantly, because there is usually only a limited number of AD weapons (effectors) available for the protection of assets. At deployment, these AD weapons are typically positioned to defend the DAs optimally in some sense.

The locations of the assets themselves, depend on a number of factors and related DA characteristics. For example, although some assets are preferably hidden within thick foliage for camouflage, radar systems require a pre-specified line of sight to perform according to the desired specification. It is evident that the specific terrain type and infrastructure are also of importance in the determination of asset locations. Of course, the positions of some DAs (such as bridges) are fixed and may hence be vulnerable to attacks. Besides asset locations, asset characteristics (or attributes) required during TE have to be determined, and a means of measuring and modelling these characteristics has to be established.

Assets may be classified into three sizes [39]: (1) point assets (up to $30 \mathrm{~m} \times 30 \mathrm{~m}$ ), (2) rectangular assets (up to $250 \mathrm{~m} \times 60 \mathrm{~m}$ ) and (3) area assets (up to $300 \mathrm{~m} \times 300 \mathrm{~m}$ ). If an asset is larger than $300 \mathrm{~m} \times 300 \mathrm{~m}$ it is usually divided into a number of smaller assets, each classified, analyzed and protected independently. Irrespective of the size of an asset, a method to interpret the asset geometry is also required. In military applications, extremely simple asset geometric modelling approaches seems to be the norm - varying sized circles, ellipses or rectangles are typically used to describe asset geometry.

Finally, although a relatively small number of DAs are usually protected during missions, priorities are typically assigned to every DA during the pre-deployment phase of a mission. These DA priorities depend on factors such as the importance of the DA to a specific mission, the repairability of the DA and the vulnerability of the DA with regards to attacks [12]. These priority values are combined with the threat value associated with a specific threat and DA, and serves as DS to the FCO (see $[33, \S 6.4]$ ) when deciding which threat to engage.

\subsection{Operators}

There are at least five operators (i.e. cognitive elements - see [33, §6.1]) either directly or indirectly involved with the TE process described in $\S 3[33, \S 6.4]$. These operators are (1) the FCO acting as operator in the loop (OIL) to the TE process; (2) the enemy operator piloting the threat (described in $\S 4.2$ ); (3) the commander responsible for coordination of enemy tactics (described in $\S 4.2 .5$ ); (4) the sensor operator manning an available sensor (described in $\S 4.3$ ); and (5) the air picture manager acting as OIL to the track management process. For the purposes of this paper, DS is tailored to focus on the FCO as an OIL for the TE process - "paying careful attention to the level, flexibility and customization of automation employed (as dictated by the tactical situation, the time available and the knowledge and situation awareness of the operator - the aim should be to aid operator decisions by execution of complex computations, flagging of suspicious aircraft behaviour or, in some cases, only to confirm operator thought)" [33, §9]. 


\section{Design of a threat evaluation decision support system}

In this section, which is the heart of the paper, we recommend a TE DSS comprising three levels of mathematical models of increasing complexity and sophistication running concurrently, with more sophisticated models being phased in as they start to produce realistic results (such as "recognising" hostile behaviour), as shown in Table 2.
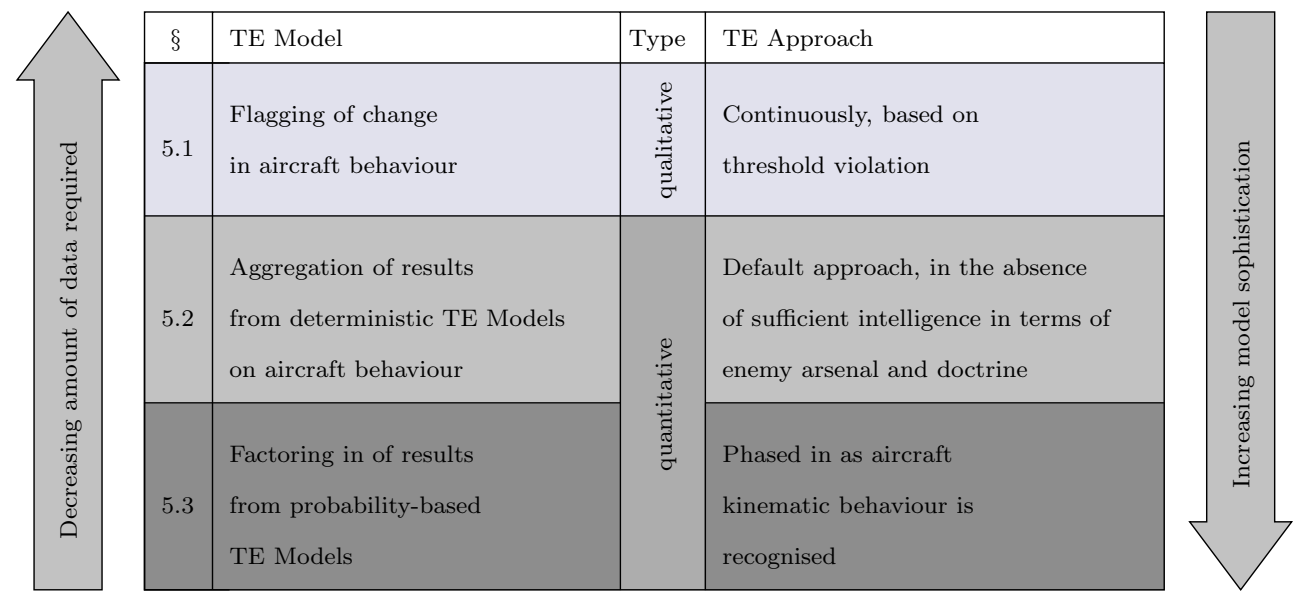

Table 2: Model hierarchy in a TE decision support system.

At the lowest level of sophistication we propose a suite of binary models, flagging aircraft for operator attention if either there is an abrupt change in their observed kinematic data or if they appear to be engaging in hostile behaviour. Although such models are not able to distinguish one aircraft as being more threatening than another, they are expected to be useful analysis tools for an operator who typically has to perform under severe stress conditions, especially when a significant number of enemy or unknown aircraft are detected. These flagging models are qualitative in nature and hence are expected to be easily implementable, as very little knowledge about the enemy arsenal or its doctrine is required for implementation purposes.

At a next level of sophistication we propose a suite of deterministic TE models, each adopting some measure of threat (such as time to DA, or some course/bearing related measure), taking observed aircraft kinematic and DA deployment data as input and producing as output a threat value within the real interval $[0,1]$. Although such models are able to distinguish one aircraft as appearing to be more threatening than another (based on the particular measure of threat) and are hence also expected to be useful analysis tools for an operator, the operator should be able to disregard or override TEs suggested by this level of models if (s)he feels justified in doing so. These deterministic models are quantitative in nature, but are still expected to be easily implementable, as very little knowledge about the enemy arsenal or its doctrine is required for implementation purposes. Very limited data in terms of own asset deployment and characteristics are also required.

At the highest level of sophistication we propose a probability-based TE model, taking aircraft kinematic data, DA deployment data, enemy arsenal intelligence and doctrine as input, and producing as output a single threat value for each aircraft with respect to each 
DA - typically an estimate of the probability that an aircraft will attack and/or kill a particular DA. Such a model will therefore also be able to distinguish one aircraft as appearing to be more threatening than another (based on the probability of attack and/or kill). This probability-based model is therefore also quantitative in nature, but is not expected to be easily implementable, because of typically considerable knowledge required about the enemy arsenal and its doctrine. However, if such information is known, then the model is expected to yield more realistic and trustworthy results than the models on lower levels of sophistication.

Our suggestion is that the above three levels of models run concurrently in the DSS, typically with the probability-based model in the background, and that some methodology be employed whereby the operator is flagged when the probability based model is able to "recognize" hostile behaviour by means of a probability of attack and/or kill for a formative element combination (aircraft, weapon to be delivered and attack technique to be used) which is significantly higher than that of other combinations. We also advocate a high level of flexibility in the DSS in the sense that an OIL should be able to configure the system output to suit his/her particular needs and analysis style. For example, one operator might wish to include only certain flagging and/or deterministic TE models in the DSS, while another may elect to disable the highest level probability based model altogether because of a lack of reliable sensor and other data $[33, \S 9]$.

Each of the three levels of models proposed above are discussed in more detail in the following subsections.

\subsection{Threat evaluation flagging models}

As mentioned above, our lowest suggested level of models to be incorporated into a TE DSS is a suite of binary models, flagging aircraft for special operator attention under certain circumstances. More specifically, we suggest two types of flagging models: models based on an absolute flagging criterion (such as when an aircraft appears to be engaging in hostile or illegal behaviour, or when its kinematic data violate fixed, pre-established bounds), and models based on a relative flagging criterion (such as when an abrupt change in the kinematic data of an aircraft is detected relative to its past behaviour).

An example of relative flagging may occur when an operator is alerted to a deviation outside a band of width some pre-established multiple of the standard deviation of an aircraft's speed around its mean speed, computed either over its entire observation history or repeatedly recomputed over a fixed-length moving time window of recent history values. Further relative flagging models may be based on other kinematic properties instead of speed, such as altitude, course, altitude change rate, turn rate, etc.

Examples ${ }^{7}$ of absolute flagging may occur (automatically, based on sensor data) when:

- an aircraft violates the air space control means ${ }^{8}$, or

\footnotetext{
${ }^{7}$ These flagging elements have been adapted from the rules of engagement in [35].

${ }^{8}$ The air space control means is violated if an aircraft enters a prohibited air zone or restricted zone without authorization, if an aircraft enters a restricted air zone from directions other than the entrance/exit gates or deviates from the AD safety lanes and tunnels, or if an aircraft flies at a forbidden speed or altitude, or in a forbidden direction.
} 
- an aircraft employs electronic counter measures.

An aircraft may additionally be flagged for operator attention based on intelligence reports whenever one or more of the following absolute flagging criteria are satisfied ${ }^{7}$ :

- if the hostility classification of the aircraft changes $^{9}$, or

- if the perceived platform type of the aircraft changes ${ }^{10}$.

The specific flagging elements incorporated into a TE DSS are of course highly dependent on sensor capability as well as on the level of optical surveillance and intelligence reporting. Because of different operator styles, it is essential for effective TE DS that an OIL should be able to configure the system (i.e. to specify which flagging elements should be included and which are to be excluded), so as not to overwhelm the operator with information deemed unnecessary by him/her.

\subsection{Deterministic threat evaluation models}

The flagging models described above are qualitative in nature; an aircraft is either flagged, based on some absolute or relative criterion, or it is not (i.e. the model outputs are binary variables). OILs may, however, require a slightly more intelligent level of TE DS hence our suggestion as to a second level of deterministic models, which are quantitative in nature. On this level we propose two suites of models, which we collectively call deterministic models: models based on temporal TE criteria capable of measuring urgency in some sense (using temporal aspects of observed aircraft kinematics relative to DA locations in order to derive an aircraft threat value, typically between 0 and 1) and models based on spatial TE criteria, capable of measuring intent in an attempt at developing early warning systems (using spatial aspects of observed aircraft kinematics in relation to DA locations in order to derive a threat value for the aircraft).

Perhaps the most commonly used deterministic model based on a temporal TE criterion is a model estimating the expected time for an aircraft to reach a specific DA. Such a model may be based on the aircraft's current speed and projected course (velocity vector), or perhaps on the assumption of a worst-case scenario where the aircraft may turn at its highest achievable turn rate until its course coincides with the asset in question and then fly towards the asset as fast as possible to deliver a weapon, as depicted in the top left of Figure 4. Aircraft associated with larger estimated times to an asset may then be assigned a smaller threat value (scaled appropriately) with respect to the asset in question than aircraft with smaller estimated times to the asset. Another example of a deterministic model based on a temporal TE criterion is a model estimating the expected time to weapon release, if an aircraft were to attack a specific DA using a particular weapon delivery technique.

\footnotetext{
${ }^{9}$ In a conventional war situation an aircraft may be classified (in a rule-based manner) as unknown or hostile if the aircraft bears enemy insignia or no insignia at all, if the aircraft is positively recognized as an enemy aircraft, if the aircraft reacts incorrectly to an identify friend/foe challenge or if the aircraft emits smoke or vapour, or drops flares or paratroopers.

${ }^{10}$ Flagging elements may be derived from non-cooperative target recognition or jet engine modulation techniques (see $\S 4.3$ ).
} 

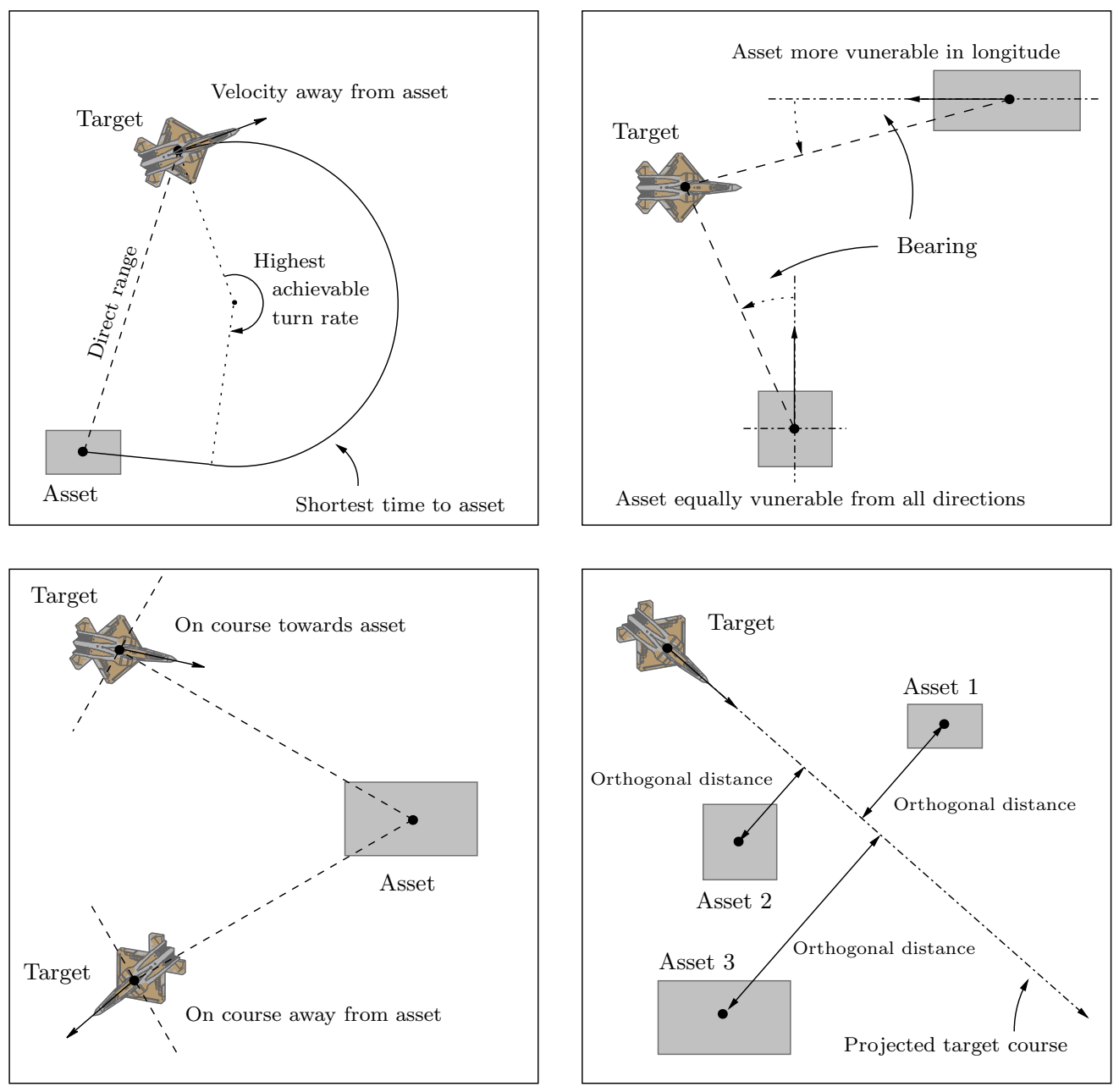

Figure 4: Deterministic TE models.

A natural spatial criterion for TE is the bearing of an aircraft with respect to a DA. The airspace of approach towards a rectangular DA may be partitioned into longitudinal and transversal approach cones (typically demarcated by the diagonals of the rectangle). An aircraft may appear more threatening for certain kinds of assets when it is detected in their longitudinal approach cones than when it is detected in their transversal approach cones (or vice versa) as shown in the top right of Figure 4, and may therefore be assigned an appropriately high or low threat value based on its observed location.

Another spatial deterministic model may base its TE of an aircraft on the degree to which the aircraft is headed towards a particular asset. If the scalar product of the course vector of an aircraft and its bearing vector with respect to the asset in question is positive, the aircraft may be said to be flying towards the asset; otherwise it may be said to be flying away from the asset, as depicted in the bottom left of Figure 4. The closer this product is to unity, the more directly the aircraft is headed towards the asset in question. If this product is non-negative (i.e. if the aircraft is flying towards the asset), one possibility would be to assign the dot product value itself as threat value to the aircraft with respect to the 
particular asset, or a zero threat value if the dot product is negative (i.e. if the aircraft is flying away from the asset). Another way of deriving a course related deterministic model of TE would be to base the threat value on the (orthogonal) pass distance from an asset if the aircraft continues along its current course, as depicted in the bottom right of Figure 4 - the shorter this pass distance, the larger the threat value (scaled appropriately) of the aircraft with respect to a particular asset.

Suppose $\mathbb{M}$ and $\overline{\mathbb{M}}$ denote respectively the sets of temporal and spatial deterministic TE models incorporated into the DSS. Then each model in $\mathbb{M} \cup \overline{\mathbb{M}}$ produces a separate threat value for each (asset, aircraft) pair at each time instant. It is natural to discretize the time continuum over which TE is performed, because information as to the tactical situation is typically received and updated at discrete points in time, as dictated by sensor refresh rates. Suppose this continuum is discretized into time intervals of a fixed length, indexed by the parameter $\tau$. The implication of such a discretization is that variables, such as the list of aerial threats as well as the kinematic attributes of each threat, are assumed constant during the time interval represented by a fixed value of $\tau$.

Suppose further that a set $\mathbb{A}(\tau)$ of DAs require protection against possible enemy aerial attacks during time interval $\tau$, and that a set $\mathbb{O}(\tau)$ of enemy aircraft are observed by means of sensor systems during that time interval. Furthermore, let $T_{j k}^{m}\left[\bar{T}_{j k}^{m}\right.$, respectively $]$ denote the threat value assigned by model $m \in \mathbb{M}[m \in \overline{\mathbb{M}}$, respectively] to aircraft $j \in \mathbb{O}(\tau)$ with respect to DA $k \in \mathbb{A}(\tau)$. Then every row in each of the threat matrices

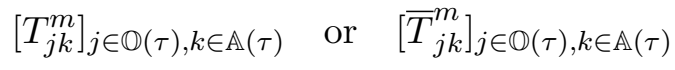

may be combined into a single threat value attributed to aircraft $j \in \mathbb{O}(\tau)$ over all DAs according to model $m$, denoted respectively by

$$
\mathcal{T}_{j}^{m}=\sum_{k \in \mathbb{A}(\tau)} V_{k} T_{j k}^{m} \quad \text { or } \quad \overline{\mathcal{T}}_{j}^{m}=\sum_{k \in \mathbb{A}(\tau)} V_{k} \bar{T}_{j k}^{m}
$$

where $V_{k} \geq 0$ denotes the relative priority of DA $k \in \mathbb{A}(\tau)$ with respect to achieving mission success, scaled so that $\sum_{k \in \mathbb{A}(\tau)} V_{k}=1$. Using the threat values in (1) a threat list $\mathbb{T}^{m}(\tau)$ or $\overline{\mathbb{T}}^{m}(\tau)$ ranking the aircraft in decreasingly threatening order with respect to all assets combined at each time instant $\tau$ may be formed according to the output of model $m \in \mathbb{M}$ or $m \in \overline{\mathbb{M}}$. These threat lists may then be presented separately to an OIL, or may be combined into OIL configured temporal or spatial threat lists by means of a weighted averaging scheme, a method of consensus ranking $[3,7,8]$ or a multiple criteria decision analysis value function method [5].

\subsection{A stochastic threat evaluation model}

It is desirable to explore, over and above the incorporation of flagging and deterministic models into a TE DSS, the development of a TE model within the domain of probability theory for two reasons: (1) a number of existing TE and WA systems reportedly reside in this domain, and (2) since probability theory is a well developed, precise and widely used mathematical discipline, in which operations and notation are well defined and interpretable, a model in this domain discourages the situation where parameter values 
measured in different units are wrongly combined (added together or subtracted from one another) - a mistake commonly made in the military domain, where decision outcomes typically depend on numerous variables with different units of measurement [33]. However, the main disadvantage of a probability-based TE model is that the quality of its results is largely based on detailed knowledge of the enemy's arsenal and doctrine - and these are not easily come by or estimated.

Denote by $P_{j, k, \tau+T}(\bullet)$ the probability that an event $\bullet$ involving aircraft $j \in \mathbb{O}(\tau)$ and asset $k \in \mathbb{A}(\tau)$ occurs during time interval $\tau+T$, where $T \geq 0$. The threat value associated with aircraft $j \in \mathbb{O}(\tau)$ with respect to asset $k \in \mathbb{A}(\tau)$ during time interval $\tau+T$ is measured in our TE model as a probability of kill of the asset in question during that time interval. A kill (denoted by $\mathcal{K}$ ) is defined as the event where an asset, because of too much damage or total destruction, loses its main functionality or purpose within a specific mission. In the case of this event the asset becomes useless to the commander and is neglected as a DA (i.e. the asset is removed from the set $\mathbb{A}(\tau))$. However, an asset that has been killed may be reclassified as a DA again (i.e. reinserted into the set $\mathbb{A}\left(\tau^{*}\right)$ during some later time interval $\tau^{*}>\tau$ ), given that repairing the DA is possible and justifiable within the time span of $\tau^{*}-\tau$ time intervals.

Of course, aircraft $j \in \mathbb{O}(\tau)$ can only inflict damage to (or kill) DA $k \in \mathbb{A}(\tau)$ during time interval $\tau+T$, if the asset is hit by a WS of the aircraft during that time interval. A hit (denoted by $\mathcal{H}$ ) is thus defined as the event of physical contact between asset and weapon. In this context the weapon may be a bullet, a missile or a fragment of a detonated warhead capable of inflicting damage. A DA can, in turn, only be hit by a WS of an aircraft if that aircraft attacks the asset with that WS, an event denoted by $\mathcal{A}$. Furthermore, the effectiveness with which an asset may be attacked depends on the formative element combination (at the very least including a specification of aircraft type, weapon type, and weapon delivery profile type) associated with an aerial threat, collectively denoted by $\mathcal{C}$. Let $\mathcal{E}$ be the event that an aircraft is hostile (i.e. an enemy aircraft), and let $\overline{\mathcal{E}}$ be the complement of this event (i.e. the event that an aircraft is friendly). If $P_{j, \tau}(\mathcal{E})$ and $P_{j, \tau}(\overline{\mathcal{E}})$ denote the probabilities with which aircraft $j \in \mathbb{O}(\tau)$ is thought to be hostile and friendly respectively during time interval $\tau$, then it follows that

$$
P_{j, k, \tau+T}(\mathcal{K})=P_{j, k, \tau+T}(\mathcal{K} \mid \mathcal{E}) P_{j, \tau}(\mathcal{E})+\underbrace{P_{j, k, \tau+T}(\mathcal{K} \mid \overline{\mathcal{E}})}_{\approx 0} P_{j, \tau}(\overline{\mathcal{E}}) .
$$

If it may be assumed that the probability of fratricide being committed (i.e. that a DA is killed by a friendly aircraft) is negligible, then it follows from (2) and the nested relation$\operatorname{ship} \mathcal{K} \subset \mathcal{H} \subset \mathcal{A}$ that

$$
\begin{aligned}
P_{j, k, \tau+T}(\mathcal{K}) & \approx P_{j, k, \tau+T}(\mathcal{A H \mathcal { K }} \mid \mathcal{E}) P_{j, \tau}(\mathcal{E}) \\
& =P_{j, k, \tau+T}(\mathcal{A H} \mathcal{H} \mathcal{E}) \\
& =\sum_{\mathcal{C} \in \mathbb{C}} P_{j, k, \tau+T}(\mathcal{A H} \mathcal{H} \mathcal{E} \mid \mathcal{C}) P_{j, k, \tau+T}(\mathcal{C}) \\
& \approx \sum_{\mathcal{C} \in \mathbb{C}} P_{j, k, \tau+T}(\mathcal{A H} \mathcal{K} \mathcal{E} \mathcal{C})
\end{aligned}
$$




$$
=\sum_{\mathcal{C} \in \mathbb{C}} \underbrace{P_{j, k, \tau+T}(\mathcal{A})}_{\text {Component I }} \underbrace{P_{j, k, \tau+T}(\mathcal{H} \mid \mathcal{A})}_{\text {Component II }} \underbrace{P_{j, k, \tau+T}(\mathcal{K} \mid \mathcal{H})}_{\text {Component III }} \underbrace{P_{j, k, \tau+T}(\mathcal{C} \mid \mathcal{K} \mathcal{E})}_{\text {Component IV }},
$$

by the multiplication rule for probabilities and by conditioning on the various formative element combinations $\mathcal{C}$, where $\mathbb{C}$ denotes the set of all possible formative element combinations at the disposal of the enemy. The approximation in (3) follows from an omission of the factor

$$
P_{j, k, \tau+T}(\mathcal{E} \mid \mathcal{A} \mathcal{H} \mathcal{K})=P_{j, k, \tau+T}(\mathcal{E} \mid \mathcal{K})=\frac{P_{j, k, \tau+T}(\mathcal{K} \mid \mathcal{E}) P_{j, \tau}(\mathcal{E})}{P_{j, k, \tau+T}(\mathcal{K} \mid \mathcal{E}) P_{j, \tau}(\mathcal{E})+\underbrace{P_{j, k, \tau+T}(\mathcal{K} \mid \overline{\mathcal{E}})}_{\approx 0} P_{j, \tau}(\overline{\mathcal{E}})} \approx 1
$$

during application of the multiplication rule, by virtue of Bayes' Theorem. Our probabilitybased TE model therefore consists of combining the results of four smaller model components in (4).

In the first model component the probability is evaluated that aircraft $j \in \mathbb{O}(\tau)$ is planning to attack DA $k \in \mathbb{A}(\tau)$ at some point $T$ time intervals into the future. The output of this model component is therefore a measure during time interval $\tau$ of the perceived current and expected future aggression in the behaviour of the particular observed aircraft.

The probability that a hit will be achieved by aircraft $j \in \mathbb{O}(\tau)$ if it attacks DA $k \in \mathbb{O}(\tau)$ during time interval $\tau+T$ is evaluated in the second model component. The output of this model component is therefore a measure during time interval $\tau$ of the expected capability of the observed aircraft within a time frame of $T$ time intervals.

In the third model component the probability is evaluated that $\mathrm{DA} k \in \mathbb{A}(\tau)$ will be damaged sufficiently to be classified as killed if hit by aircraft $j \in \mathbb{O}(\tau)$ when attacking the asset during time interval $\tau+T$. The output of this model component is therefore a measure during time interval $\tau$ of the vulnerability of the particular asset with respect to the threat posed by the observed aircraft if attacked $T$ time intervals into the future by that aircraft.

Finally, the probability that a particular formative element combination will have been utilised by the enemy in order to achieve a kill of DA $k \in \mathbb{A}(\tau)$ by observed aircraft $j \in \mathbb{O}(\tau)$ at time $\tau+T$ is evaluated in the fourth model component. The output of this final model component is therefore a measure at time $\tau$ of the suitability of an enemy formative element combination with respect to being capable of killing a specific DA within $T$ time units.

Note that both intent and capability are captured by the model in (4) [33]. Possible approaches towards estimating the four probabilities in the various model components are now described separately in more detail.

\subsubsection{Model component I, $P_{j, k, \tau+T}(\mathcal{A})$}

Since our perception of the aggression inherent in the behaviour of aircraft $j \in \mathbb{O}(\tau)$ is based on its classification as unknown/hostile or friendly and on the formative elements combined in that observation, it is natural to condition on these dependencies to obtain an expression 


$$
\begin{aligned}
P_{j, k, \tau+T}(\mathcal{A}) & =P_{j, k, \tau+T}(\mathcal{A} \mid \mathcal{E}) P_{j, \tau}(\mathcal{E})+\overbrace{P_{j, k, \tau+T}(\mathcal{A} \mid \overline{\mathcal{E}})}^{\approx 0} P_{j, \tau}(\overline{\mathcal{E}}) \\
& \approx P_{j, \tau}(\mathcal{E}) \sum_{\mathcal{C} \in \mathbb{C}} P_{j, k, \tau+T}(\mathcal{A} \mid \mathcal{E} \mathcal{C}) P_{j, k, \tau+T}(\mathcal{C} \mid \mathcal{E})
\end{aligned}
$$

for the probability that aircraft $j \in \mathbb{O}(\tau)$ will attack asset $k \in \mathbb{A}(\tau)$ during time interval $\tau+T$. The probability $P_{j, k, \tau+T}(\mathcal{C} \mid \mathcal{E}) \approx P_{j, k, \tau}(\mathcal{C} \mid \mathcal{E})$ in $(5)$ that a specific formative element combination $\mathcal{C} \in \mathbb{C}$ chosen by the enemy is embodied in aircraft $j \in \mathbb{O}(\tau)$ depends on the enemy's arsenal, and may, as a point of departure, be estimated by means of a combination of expert opinion and pre-deployment intelligence reports. However, a better approach may be to base a likelihood estimation $[1,34]$ that an enemy aircraft $j \in \mathbb{O}(\tau)$ represents a specific formative element combination $\mathcal{C} \in \mathbb{C}$ on the kinematic history values associated with the aircraft relative to the expected values of these attributes. These expected values may, in turn, be derived from past observations and expert opinion if the latter are documented and updated in a tactical database of past observations of formative element combination attribute values and bounds.

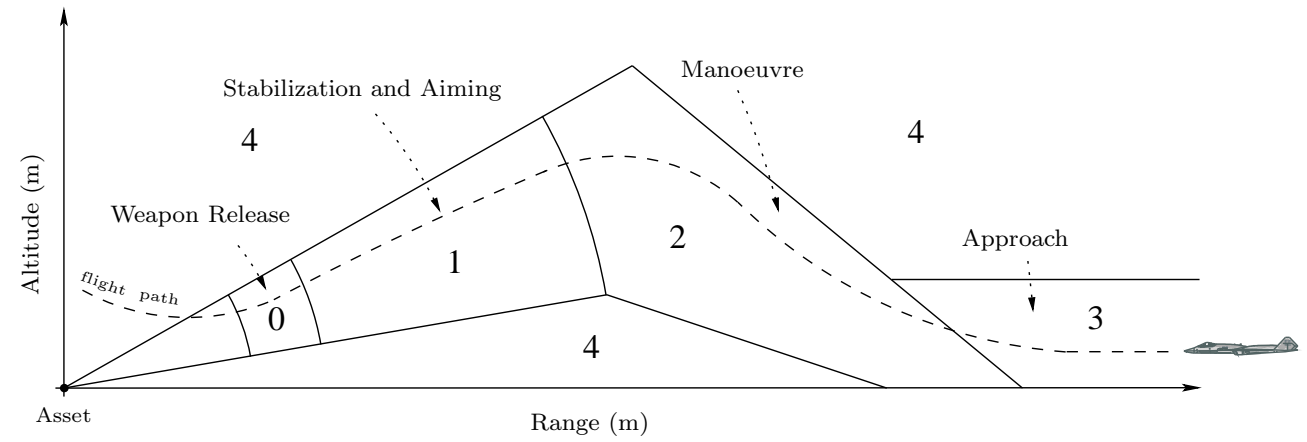

Figure 5: Weapon delivery stages $\mathbb{S}_{\mathcal{C}^{*}}=\{0,1,2,3,4\}$ associated with a hypothetical formative element combination $\mathcal{C}^{*} \in \mathbb{C}$.

In order to describe a method for estimating the probability $P_{j, k, \tau+T}(\mathcal{A} \mid \mathcal{E C})$ in $(5)$ a means is typically required by which the progression of an aircraft through the various stages of delivering a weapon to a DA using some attack technique may be modelled mathematically. Suppose that delivery of a weapon in enemy formative element combination $\mathcal{C} \in \mathbb{C}$ comprises the $n_{\mathcal{C}}+1$ stages $\mathbb{S}_{\mathcal{C}}=\left\{0,1,2, \ldots, n_{\mathcal{C}}-1, n_{\mathcal{C}}\right\}$ sequenced in decreasing order during execution of the technique - stages 0 and $n_{\mathcal{C}}$ denoting respectively the weapon release stage (i.e. the stage during which the actual attack takes place) and a generic stage representing unknown aircraft behaviour (see Figure 5 for an example). If $\mathcal{S}_{\mathcal{C}, i}$ denotes the event that formative element $\mathcal{C} \in \mathbb{C}$ is in stage $i \in \mathbb{S}_{\mathcal{C}}$ of weapon delivery and if $T_{\mathcal{C}, i}$ denotes the estimated number of time steps required for formative element combination $\mathcal{C} \in \mathbb{C}$ to progress from stage $i$ to stage 0 of weapon delivery, then the probability $P_{j, k, \tau+T}(\mathcal{A} \mid \mathcal{E C})$ in (5) may be written as

$$
P_{j, k, \tau+T}(\mathcal{A} \mid \mathcal{E C})=\sum_{i \in \mathbb{S}_{\mathcal{C}}} P_{j, k, \tau}\left(T_{\mathcal{C}, i}=T\right) P_{j, k, \tau}\left(\mathcal{S}_{\mathcal{C}, i}\right)
$$


by conditioning on the stage of weapon delivery in which the formative element combination finds itself during time interval $\tau$. Both probabilities on the right hand side of (6) may be estimated, based on the current and past kinematic properties of aircraft $j \in \mathbb{O}(\tau)$. A tool capable of estimating future kinematic attributes of an aircraft (such as speed, position and course) is additionally required for the evaluation of the probability $P_{j, k, \tau}\left(T_{\mathcal{C}, i}=T\right)$ in $(6)$ - perhaps by backtracking from where an aircraft is expected to be during stage 0 of the attack profile according to current aircraft kinematic attributes, so as to determine to what degree it currently possesses the desired kinematic attributes required to affect a stage progression from $i$ to 0 within $T$ time intervals.

\subsubsection{Model component II, $P_{j, k, \tau+T}(\mathcal{H} \mid \mathcal{A})$}

The probability that a weapon delivered by aircraft $j \in \mathbb{O}(\tau)$ hits asset $k \in \mathbb{A}(\tau)$ during time interval $\tau+T$,

$$
\begin{aligned}
P_{j, k, \tau+T}(\mathcal{H} \mid \mathcal{A}) & =P_{j, k, \tau+T}(\mathcal{H} \mid \mathcal{E} \mathcal{A}) \overbrace{P_{j, k, \tau+T}(\mathcal{E} \mid \mathcal{A})}^{\approx 1}+P_{j, k, \tau+T}(\mathcal{H} \mid \overline{\mathcal{E}} \mathcal{A}) \overbrace{P_{j, k, \tau+T}(\overline{\mathcal{E}} \mid \mathcal{A})}^{\approx 0} \\
& \approx \sum_{\mathcal{C} \in \mathbb{C}} P_{j, k, \tau+T}(\mathcal{H} \mid \mathcal{E} \mathcal{A C}) P_{j, k, \tau+T}(\mathcal{C} \mid \mathcal{E} \mathcal{A})
\end{aligned}
$$

may be evaluated by first conditioning on whether the aircraft is thought to be an enemy craft and then conditioning on the formative element combinations of the enemy.

The probability $P_{j, k, \tau+T}(\mathcal{H} \mid \mathcal{E} \mathcal{A C})$ in $(7)$ may, as a first order approach, be estimated by a combination of expert opinion and pre-deployment intelligence reports. Since the value of $P_{j, k, \tau+T}(\mathcal{H} \mid \mathcal{E} \mathcal{A C})$ predominantly depends on the hit probability of the weapon delivered by the enemy, this value is typically supplied by the weapon manufacturer - given certain conditions or thresholds regarding the tactical environment in which the weapon operates and the way in which the weapon is delivered. A better, but more complex, approximation of this value may be obtained by investigating the observed aircraft's deviation from the attack technique (embodied in $\mathcal{C} \in \mathbb{C}$ ) over time $T$ or by modelling the effectiveness of the weapon given the current weather conditions during the time window investigated.

Finally, the probability $P_{j, k, \tau+T}(\mathcal{C} \mid \mathcal{E} \mathcal{A})$ in $(7)$ may be evaluated by means of a data mining technique $[1,21,34]$, based on the enemy's arsenal and known templates of enemy attack profiles.

\subsubsection{Model component III, $P_{j, k, \tau+T}(\mathcal{K} \mid \mathcal{H})$}

The probability that an aircraft $j \in \mathbb{O}(\tau)$ kills DA $k \in \mathbb{A}(\tau)$ during time interval $\tau+T$,

$$
\begin{aligned}
P_{j, k, \tau+T}(\mathcal{K} \mid \mathcal{H}) & =P_{j, k, \tau+T}(\mathcal{K} \mid \mathcal{E} \mathcal{H}) \overbrace{P_{j, k, \tau+T}(\mathcal{E} \mid \mathcal{H})}^{\approx 1}+P_{j, k, \tau+T}(\mathcal{K} \mid \overline{\mathcal{E}} \mathcal{H}) \overbrace{P_{j, k, \tau+T}(\overline{\mathcal{E}} \mid \mathcal{H})}^{\approx 0} \\
& \approx \sum_{\mathcal{C} \in \mathbb{C}} P_{j, k, \tau+T}(\mathcal{K} \mid \mathcal{E} \mathcal{H C}) P_{j, k, \tau+T}(\mathcal{C} \mid \mathcal{E} \mathcal{H})
\end{aligned}
$$

may similarly be evaluated by first conditioning on whether the aircraft is thought to be an enemy craft and then conditioning on the formative element combinations of the enemy. 
The probability $P_{j, k, \tau+T}(\mathcal{C} \mid \mathcal{E} \mathcal{H})$ may be estimated in a manner similar to the estimation of $P_{j, k, \tau+T}(\mathcal{C} \mid \mathcal{E} \mathcal{A})$ in the previous model component. Finally, the probability $P_{j, k, \tau+T}(\mathcal{K} \mid \mathcal{E} \mathcal{H C})$ in (8) may, as a point of departure, be estimated by a combination of expert opinion and pre-deployment (mobilisation and appreciation) intelligence reports. The value of $P_{j, k, \tau+T}(\mathcal{K} \mid \mathcal{E} \mathcal{H C})$ depends on the hit probability of the weapon delivered by the enemy and on the characteristics of the asset being attacked.

\subsubsection{Model component IV, $P_{j, k, \tau+T}(\mathcal{C} \mid \mathcal{K} \mathcal{E})$}

Finally, the probability $P_{j, k, \tau+T}(\mathcal{C} \mid \mathcal{K} \mathcal{E}) \approx P_{j, k, \tau}(\mathcal{C} \mid \mathcal{K} \mathcal{E})$, if DA $k$ were to be killed, that a specific combination of enemy formative elements is embodied in observed aircraft $j \in$ $\mathbb{O}(\tau)$, may also be estimated by a combination of expert opinion and pre-deployment intelligence reports.

\section{Implementation thoughts}

The purpose of this section is to provide the reader with some thoughts regarding the implementation of a TE subsystem as part of a TEWA system. However, the ideas put forward here are by no means exhaustive and should be appreciated merely as a stepping stone towards implementation of a proper TE system architecture.

Consider Figure 6, which shows various processes internal to a TEWA system (such as TE, WA and attribute management processes), a TEWA database, various processes external to a TEWA system but influencing it (such as FCO DS, a track management process and a maintenance process), as well as the data flow between these processes. Note that the term process may refer to separate applications or to functional blocks of code within a single software TEWA application. The dataflow and functioning of the processes in Figure 6 are discussed in more detail below, with special reference to the data structures related to the TE process within the TEWA database shown schematically in Figure 7.

The main TEWA process is not shown as a single entity in Figure 6. For the purposes of this paper, we assume that the main process is capable of receiving, processing and storing data from external sources, distributing data to the relevant sub-processes (e.g. the TE process) and controlling the sequence in which processes are run.

During pre-deployment, we propose that maintenance processes (see Figure 6, bottom left) should be used to update the initialization parameters of the various TE Models (i.e. the flagging ( 55.1$)$, deterministic ( $\$ 5.2)$ and probability-based ( 55.3$)$ models). A set of initialization parameters may thus be stored for every TE Model (see Figure 7, middle and bottom). Examples of these parameters may include the aircraft, weapon and technique types of the enemy arsenal and their related attack profile templates ${ }^{11}$, as well as the attribute thresholds for the flagging models.

During deployment various attributes related to sensors and DAs (e.g. their positions and

\footnotetext{
${ }^{11}$ As mentioned before, an understanding of enemy doctrine and capabilities may be a very cumbersome process, since behaviour regarding attack techniques and force movement strategies are typically kept secret $(\operatorname{see} \S 2)$.
} 


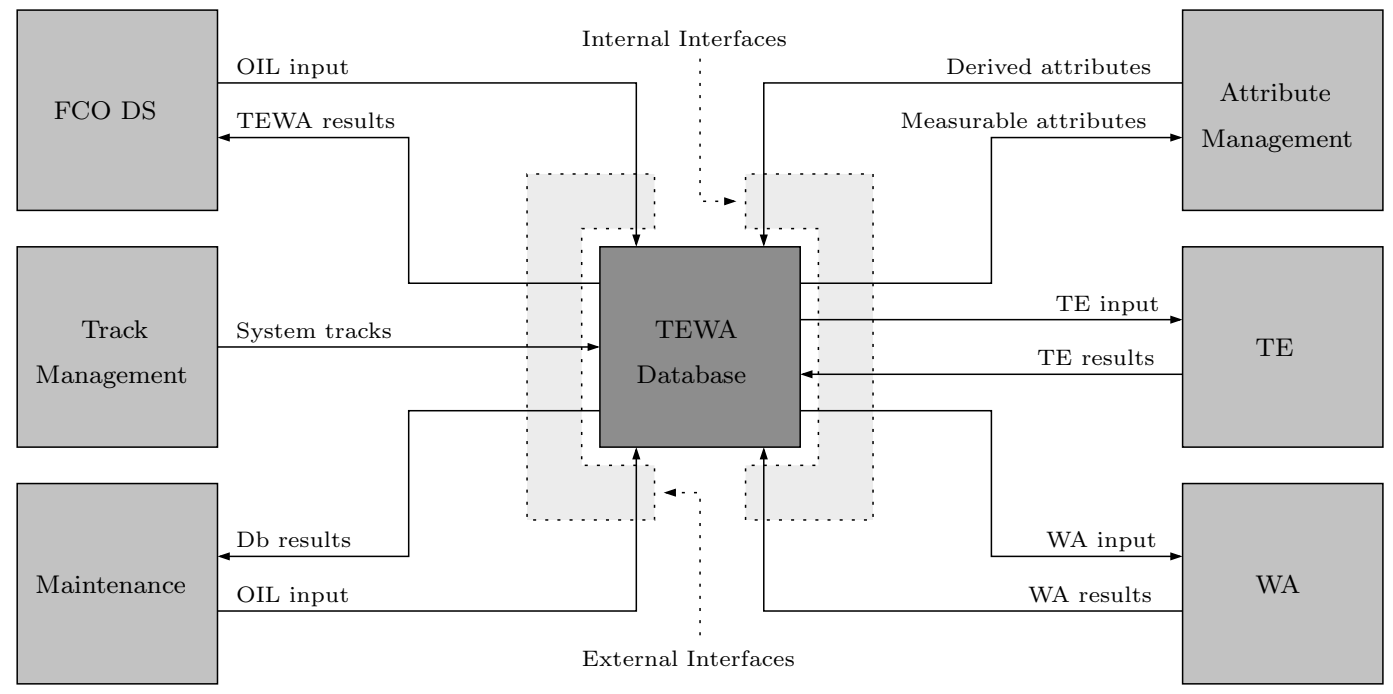

Figure 6: Dataflow between the internal processes, external processes and the database of a TEWA system.

priorities) are typically determined and should also be stored in the database (see Figure 7 , top right). In order to achieve flexibility, these attributes should be stored as system tracks and should be updated in real-time (e.g. when the position of a mobile sensor system changes), similar to the system tracks of targets. Although flexibility is thus improved, this approach is expected to increase the processing power required for TEWA processes. For example, if the position of a sensor system changes, a number of off-line computations (such as LOS computations) previously performed during the pre-deployment phase of the mission have to be repeated in real-time.

The set of measured attributes available is determined by the deployment of the sensor systems (see §4.3) and the set of derived attributes is subsequently computed from the set of available measured attributes. TE models may require specific input parameters (i.e. certain combinations of measured and derived attributes) and hence the sensor systems also dictate which TE models are potentially available during a specific tactical situation.

Let us assume, for the purposes of this paper, that a TEWA computation cycle is triggered either by the change of a data field or set entry in the database (e.g. target, sensor or DA system track changes) or by an event fired periodically at every implementation clock cycle. The choice of a suitable trigger depends on a number of factors, including the time a TE model requires for completion of its computations, the rate at which data are required from a DS perspective and the rate at which observed sensor data are updated (see $\S 4.3$ ). The trigger signals the start of a sequential TEWA process during which threat attributes are computed, threats are evaluated and available effectors are assigned to engage these threats. This sequential TEWA process is repeated during each TEWA computation cycle.

When a TEWA computation cycle is triggered, the system should effectively take a snapshot of the database at that specific time instant. The available measured attributes, observed by the sensor systems and fused by a track management processes (see Figure 6 , middle left), should then be sent to an attribute manager (i.e. the implementation of 


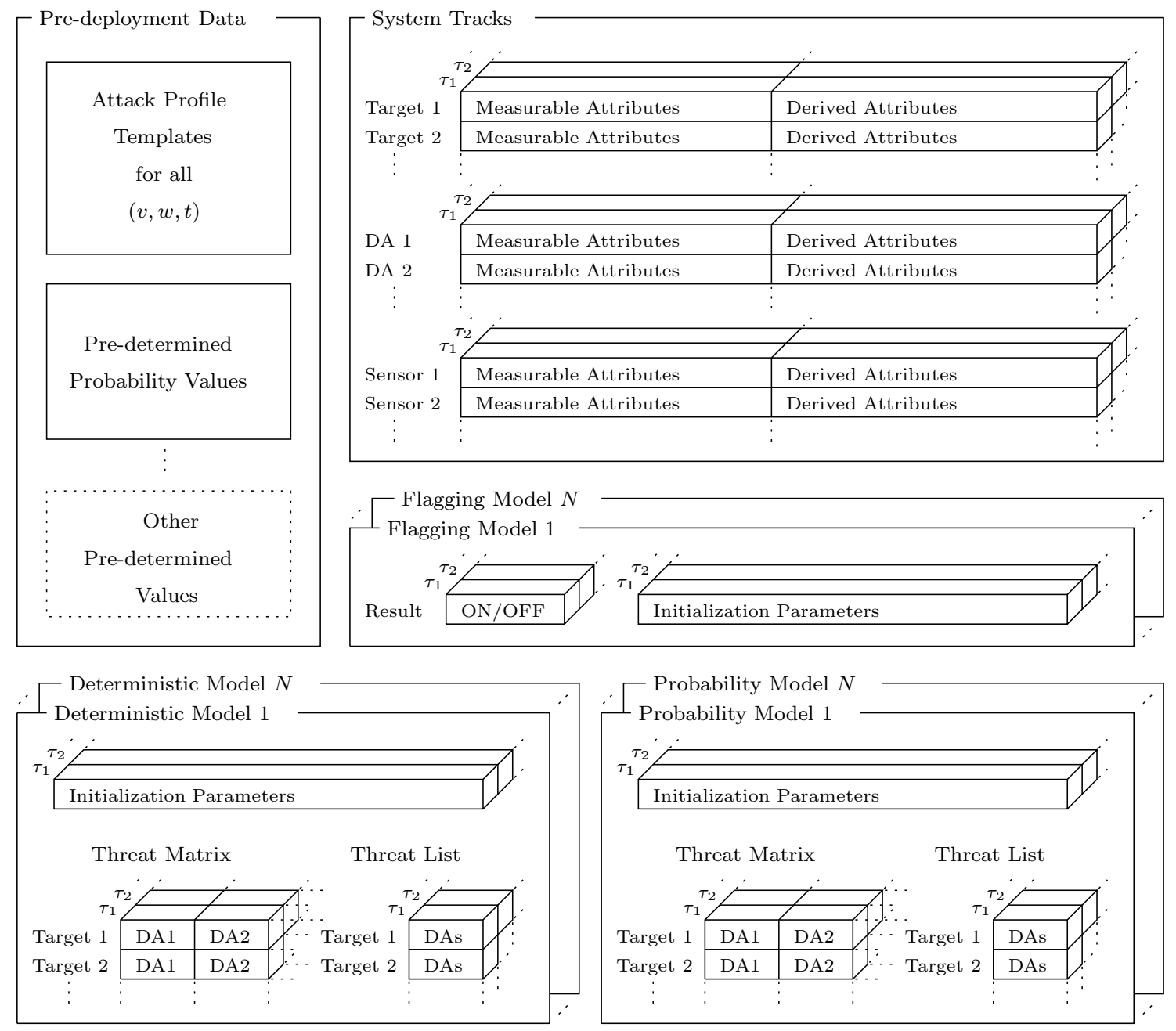

Figure 7: The TEWA database with focus on the TE-related datastructures.

an attribute management processes in Figure 6, top right) where derived attributes are computed. The derived attributes of every observed threat should then be stored in the database as part of the target's system track at that time instant (see Figure 7, top right). Thresholds may be set with regards to the required quality or quantity of data. For example, if the quality of a specific measured attribute (e.g. altitude) is deemed insufficient, related derived attributes and TE models requiring that measured attribute may not be available during that TEWA computation cycle.

After completing the process of attribute management, the TE process should be notified to commence operation. Every available TE model (i.e. flagging, deterministic and probability-based models) should receive the measured attributes, derived attributes, and pre-deployment data required, as well as relevant initialisation parameters as customized by the OIL (during pre-deployment or in real-time). Flagging models will produce a binary result (either on or off), whereas the deterministic and probability-based models will result in a threat matrix (containing the threat value of every DA/threat pair) and 
a prioritised list of threats as explained in $§ 5.2$. All these threat lists should then be combined into one threat list according to some (multi-criteria decision analysis) value function method, representing prioritised threats with respect to all the DAs combined.

Although the TE models that may potentially be used during a TEWA computation cycle are determined by pre-deployment data (e.g. sensors available) and real-time data (e.g. the quality of data), the OIL should also be able to configure those TE models actually used. That is, operators should be able choose which TE models to use for TE purposes. Proper DS user interfaces and result visualisation are vital, since the OIL may easily be overwhelmed by the amount and update rate of the data produced [33].

All TE results should be stored in the database (see Figure 7, middle and bottom) after which the WA system should be notified to commence operation. The WA process is the final process of the TEWA computation cycle during which feasible engagements between effectors and threats are proposed (based on the combined, prioritised threat list). Finally the OIL should be responsible for confirming or ordering actual engagements in order to protect the DAs in his/her area of responsibility. Thereafter the next TEWA computation cycle commences.

\section{Conclusion}

In this paper we identified the typical elements of a TE DSS in a GBADS environment (in §4) and we suggested an approach towards such a TE DSS comprising three levels of mathematical models of increasing complexity and sophistication running concurrently, with more sophisticated models being phased in as they start to produce realistic results (see $\S 5)$. At the lowest level of sophistication we proposed a suite of binary (qualitative) models (§5.1), flagging aircraft for operator attention if either there is an abrupt change in their kinematic data or if they appear to be engaging in hostile behaviour. At a next level of sophistication we proposed a suite of deterministic (quantitative) TE models ( $\$ 5.2$ ), each adopting some measure of threat (such as time to asset, or some course/bearing related measure), taking aircraft kinematic and DA deployment data as input and producing as output a scaled, real threat value. At the highest level of sophistication we proposed a probability-based (quantitative) TE model ( $(5.3)$, taking aircraft kinematic data, DA deployment data and enemy arsenal intelligence as well as doctrine as input and producing as output a single value, typically an estimate of the probability that an aircraft will attack and/or kill a particular DA. We also put forward some thoughts regarding the implementation of a TE subsystem as part of a TEWA system in $\S 6$.

The challenges facing a developer of a TEWA system as we have outlined in this paper are certainly non-trivial, but perhaps the most significant challenge faced by such a developer is to deliver a sellable product. In order to achieve the goal of delivering a sellable product, TE system output is required to confirm operator thought and to follow an OIL approach. This problem is exacerbated in the military context where operators are often very set in their ways, where their methods of TE typically differ significantly and where the majority of South African operators have only very recently been introduced to advanced computerised DSSs. 
The focus of a TE system should be to operate as a DSS providing TE results and various derived aircraft attributes to an $\mathrm{AD}$ control operator that are typically too tedious to compute by hand. A TE system developer should not seek to deliver a product capable of replacing or outperforming a human operator — such a goal is unattainable (due to the complex thought processes and cumulative experience embodied in a human operator) and would be disastrous if attempted (in terms of the public relations exercise of selling the product).

\section{Acknowledgements}

The authors are especially indebted to Mr Bob Visser and Mr Pieter-Jan Wolfaardt (RRS), Dr Jan Roodt and Mr Cobus Nel (CSIR-DPSS), and Mr René Oosthuizen (Monzé Consultants) for many fruitful discussions and for making the facilities (libraries and software) at their institutions available to the authors. The authors are also indebted to Mr Hanlo Pretorius, Col André Greef, Lt Col Jacques Baird, Srg WO1 Piet Gouws, Lt Col Labuschagne, Col Niek du Plessis, Lt Col Marchand Mostert, Maj Lance Wellington and Maj Jannie Scott, all from the SANDF, for valuable end-user input. We further thank Mr Peet Fourie and Mr Sven Holfelder (RRS), as well as Mr Schalk Verwey (Denel), for their willingness to share considerable expertise in the military industry with us. Ms Anita Louis, Mr Herman le Roux, Mr Shahen Naidoo and Mr Bernardt Duvenhage (all from CSIR-DPSS) as well as Ms Martie Muller, Mr Danie Bence and Mr Leon Downes (from IMT) are thanked for valuable inputs with respect to real-time TEWA simulation and testing. $\mathrm{Mr}$ Gerhardt Strutters and Mr Daniel Seegmuller (TFDC), Mr Johan Badenhorst (Epsilon) and Mr Jan Durand (ARMSCOR) are thanked for their efforts to make real test data available to the authors. Research towards this paper was funded by the Department of Defence via ARMSCOR Contract KT435271 and by the National Research Foundation (GUN 2072999). Finally, the authors are grateful to Mr Johan Mostert (ARMSCOR) and Col Tammy Mdekazi (SANDF) for facilitating contact with operators and experts within the SANDF.

\section{References}

[1] Balakrishnan N, 1992, Handbook of the logistic distribution, Marcel Dekker Inc., New York (NY).

[2] BBC History, 2006, Industrialisation, [Online], [Cited 2007, August 20th], Available from: http://www.bbc.co.uk/history/

[3] Beck MP \& Lin BW, 1983, Some heuristics for the consensus ranking problem, Computers and Operations Research, 10(1), pp. 1-7.

[4] Bell MR \& GRubBs RA, 1993, JEM modeling and measurement for radar target identification, Aerospace and Electronic Systems, 29(1), pp. 73-87.

[5] Belton V \& Stewart TJ, 2002, Multiple criteria decision analysis: An integrated approach, Kluwer Academic Publishers, Boston (MA).

[6] Cebrowski AK, 2005, Network centric warfare and information superiority, [Online], [Cited 2007, August 20th], Available from: http://www.oft.osd.mil/

[7] Cook WD, 2006, Distance-based and ad hoc consensus models in ordinal preference ranking, European Journal of Operational Research, 172, pp. 369-385. 
[8] Cook WD \& SeIford LM, 1978, Priority ranking and consensus formation, Management Science, 24(16), pp. 1721-1732.

[9] CSIR, 2005, Non-cooperative target recognition for search and track radars, [Online], [Cited 2007, August 20th], Available from: http://www.csir.co.za/

[10] Defence R\&D CAnada, 2007, Non-cooperative target recognition of air targets, [Online], [Cited 2007, August 20th], Available from: http:// www.ottawa.drdc-rddc.gc.ca/

[11] Duffy M, 2007, First world war, [Online], [Cited 2007, August 20th], Available from: http://www.firstworldwar.com/

[12] Du Toit FJ, 2006, Design of an automated weapons assignment system, Final Year Applied Mathematics Project, Department of Mathematical Sciences, University of Stellenbosch, Stellenbosch.

[13] The Guardian, 2001, Fourth generation warfare, [Online], [Cited 2007, August 20th], Available from: http://publish.uwo.ca/

[14] Harris B, 2007, Mao Zedong, [Online], [Cited 2007, August 20th], Available from: http://www.moreorless.au.com/

[15] Ho CK, Robinson A, Miller DR \& Davis MJ, 2005, Overview of sensors and needs for environmental monitoring, Sensors, 5, pp. 4-37.

[16] Hogg I, 1986, The weapons that changed the world, Edury Press, London.

[17] HowARD SP, 2000, Special operations forces and unmanned aerial vehicles: Sooner or later?, [Online], [Cited 2007, August 20th], Available from: http:// www.specialoperations.com/Focus/UAVs.htm

[18] Hutchins SG, Kelly RT \& Morrison JG, 1996, Decision making for tactical decision making under stress, Naval Postgraduate School, Monterey (CA).

[19] Interstate Statistical Committee of the Commonwealth of Independent States, 2007, About Commonwealth of Independent States, [Online], [Cited 2007, August 20th], Available from: http://www.cisstat.com/eng/cis.htm

[20] JUAV-JTE, 2004, Joint unmanned aerial vehicles joint test and evaluation, [Online], [Cited 2004, August 9th], Available from: http://www.juav.jte.osd.mil/

[21] Larose DT, 2005, Discovering knowledge in data: an introduction to data mining, John Wiley \& Sons, Inc, Englewood Cliffs, New Jersey (NJ).

[22] Liebhader MJ \& Smith CAP, 1997, Naval air defense threat assessment: Cognative factors and model, Pacific Science \& Engineering Group, San Diego (CA).

[23] Kirby MW, 2003, Operational research in war and peace, Imperial College Press, London.

[24] Machinebrain.com, 2007, Fighting robots: Military robots, [Online], [Cited 2007, August 20th], Available from: http://www.machinebrain.com/

[25] Mulvenon JC \& YAng RH, 2007, The People's Liberation Army in the information age, [Online], [Cited 2007, August 20th], Available from: http://www.rand.org/

[26] National Air and Space Museum, 2007, Inventing a flying machine, [Online], [Cited 2007, August 20th], Available from: http://www.nasm.si.edu/

[27] North Park University, 1997, The first Balkan war, [Online], [Cited 2007, August 20th], Available from: http://www. thenagain.info/

[28] On WAR, 2007, Armed conflict events data, [Online], [Cited 2007, August 20th], Available from: http://www. onwar.com/aced/data/

[29] PARK WJ, 2007, UAV Center, [Online], [Cited 2007, August 20th] Available from: http://www . uavcenter.com/

[30] Raduege HD, 2004, Net-centric warfare is changing the battlefield environment, [Online], [Cited 2007, August 20th], Available from: http://www.stsc.hill.af.mil/

[31] Ross S, 2006, A first course in probability, $7^{\text {th }}$ Edition, Pearson/Prentice Hall, Upper Saddle River (NJ).

[32] Roux JN, 2005, Real-time threat evaluation of fixed wing aircraft in a ground based air defence environment, MScEng Thesis, University of Stellenbosch, Stellenbosch.

[33] Roux JN \& VAn Vuuren JH, 2007, Threat evaluation and weapon assignment decision support: A review of the state of the art, ORiON, 23(2), pp. 151-187.

[34] Silverman BW, 1990, Density estimation for statistics and data analysis, Chapman \& Hall, London. 
[35] South African air defence artillery doctrine handbook, Volume 2, ARMSCOR, Pretoria, 2002.

[36] Schevill F, 1966, History of the Balkan Peninsula, Frederick Ungar Publishing Inc, New York (NY).

[37] STEIn FP, 2006, Observations on the emergence of network centric warfare, [Online], [Cited 2007, August 20th], Available from http://www.dodccrp.org/

[38] Tzu S, 2004, The art of war, [Online], [Cited 2007, August 20th], Available from: http://www.druglibrary .org/schaffer/general/artof war/index.htm

[39] UDDC, 2006, Asset assessment, (Unpublished) Doctrinal Note, Volume 2: Book 2: Pamphlet 2 (Restricted), UDDC, Kimberley.

[40] VeST J, 2001, Fourth-generation warfare, [Online], [Cited 2007, August 20th], Available from: http://www. theatlantic.com/

[41] Viegas J, 2004, New combat robot prepares for duty, [Online], [Cited 2007, August 20th], Available from: http://dsc.discovery.com/

[42] Visser B, Military Expert, Reutech Radar Systems, Personal Communications, 2004-2007, Contactable at: bvisser@rrs.co.za

[43] Wikipedia: The free encyclopedia, 2007, Doppler effect, [Online], [Cited 2007, August 20th], Available from: http://en.wikipedia.org/wiki/Doppler_effect

[44] Wikipedia: The Free EnCyClopedia, 2007, Inverse synthetic aperture radar (ISAR), [Online], [Cited 2007, August 20th], Available from: http://en.wikipedia.org/wiki/Inverse_synthetic _aperture_radar

[45] Wikipedia: The free encyclopedia, 2007, Military science, [Online], [Cited 2007, August 20th], Available from: http://en.wikipedia.org/wiki/Military_science

[46] Wikipedia: The free encyclopedia, 2007, Radar, [Online], [Cited 2007, August 20th], Available from: http://en.wikipedia.org/wiki/Radar

[47] Wikipedia: The free encyclopedia, 2007, Sensor, [Online], [Cited 2007, August 20th], Available from: http://en.wikipedia.org/wiki/Sensor

[48] Wyatt E \& Thrasher R, 2005, UCAV transitioned to J-UCAS, [Online], [Cited 2007, August 20th], Available from: http://www.darpa.mil/tto/programs/ucav.html

[49] White FE, 1988, A model for data fusion, Proceedings of the 1st National Symposium on Sensor Fusion, Volume 2, Orlando (FL).

[50] Wolfandit PJ, Technology Executive, Reutech Radar Systems, Personal Communications, 20042007, Contactable at: pjwolf@rrs.co.za

\title{
Appendix
}

\author{
AD Air Defence \\ DA Defended Asset \\ DS Decision Support \\ DSS Decision Support System \\ FCO Fire Control Officer \\ GBADS Ground Based Air Defence System \\ NCW Network Centric Warfare \\ OIL Operator in the Loop \\ TE Threat Evaluation \\ TEWA Threat Evaluation \& Weapon Assignment \\ WS Weapon System
}


